\title{
Integration of high-throughput data of microRNA and mRNA expression profiles reveals novel insights into the mechanism of liver fibrosis
}

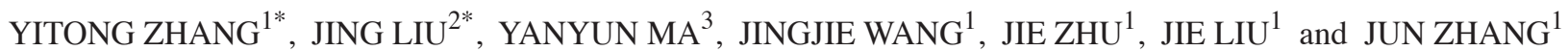 \\ ${ }^{1}$ Department of Digestive Diseases, Huashan Hospital, Fudan University, Shanghai 200040; \\ ${ }^{2}$ State Key Laboratory of Genetic Engineering, Collaborative Innovation Center for Genetics and Development; \\ ${ }^{3}$ Ministry of Education Key Laboratory of Contemporary Anthropology, Department of Anthropology and Human Genetics, \\ School of Life Sciences, Fudan University, Shanghai 200438, P.R. China
}

Received April 4, 2018; Accepted October 9, 2018

DOI: $10.3892 / \mathrm{mmr} .2018 .9641$

\begin{abstract}
Numerous studies have revealed that microRNAs (miRNAs) are functional non-coding RNAs that serve roles in a variety of biological processes. However, the expression patterns and regulatory networks, as well as the miRNAs involved in liver fibrosis remain to be elucidated. In the present study, a mouse model of liver fibrosis was constructed by $\mathrm{CCl}_{4}$ intraperitoneal injection and the total RNAs were extracted from the liver of the mice. The total RNAs were then sequenced on an Illumina HiSeq 2000 platform and an integrated analysis of miRNA and mRNA expression profiles in $\mathrm{CCl}_{4}$-induced liver fibrosis was performed. Compared with normal liver samples, 56 and 15 miRNAs were found to be upregulated and downregulated in fibrotic livers, respectively. To predict the potential functions of these miRNAs, bioinformatics analysis, including Gene Ontology and Kyoto Encyclopedia of Genes and Genomes pathway analysis, was used to assess target mRNAs. The results indicated that the mitogen-activated protein kinase, phosphoinositide 3 kinase/protein kinase B and focal adhesion signaling pathways were the most significantly enriched. In addition, a regulatory network containing five dysregulated miRNAs and 22 target mRNAs was constructed based on their inverse correlation. Furthermore, the five dysregulated miRNAs were significantly upregulated and the expression of RELB, RAP1A, PPP3CB, MAP2K4, ARRB1, MAP3K4, FGF1 and PRKCB in the network was significantly decreased in LX-2 cells following TGF- $\beta 1$ treatment which
\end{abstract}

Correspondence to: Dr Jun Zhang, Department of Digestive Diseases, Huashan Hospital, Fudan University, 12 Middle Wulumuqi Road, Shanghai 200040, P.R. China

E-mail: archsteed@gmail.com

${ }^{*}$ Contributed equally

Key words: liver fibrosis, microRNA expression, microRNA-mRNA network suggested that they were associated with the activation of human hepatic stellate cells. The miRNA-mRNA regulatory network produced in the present study may provide novel insights into the role of miRNAs in liver fibrosis.

\section{Introduction}

Hepatic fibrosis is an inevitable process that occurs in the progression of various chronic liver diseases, ultimately leading to cirrhosis. It is a reversible pathological change caused by acute/chronic liver injury (1). Liver fibrosis manifests as the excessive deposition of extracellular matrix (ECM) in the liver. Long-term aggravation of liver fibrosis results in diffuse liver damage, which may lead to the development of cirrhosis and liver cancer (1). The activation and proliferation of hepatic stellate cells (HSCs) is critically involved in the process of liver fibrosis (2-4). It is therefore of great importance to identify a reliable, non-invasive and convenient method for the early diagnosis of liver fibrosis.

MicroRNAs (miRNAs/miRs) are a class of evolutionarily conserved small (18-24 nucleotides) single-stranded non-coding RNAs that mediate post-transcriptional gene suppression, via the degradation of target mRNAs or the suppression of mRNA translation following binding $(5,6)$. Increasing evidence suggests that dysregulated miRNAs serve a crucial role in a number of diseases, including hepatocellular carcinoma and liver fibrosis $(7,8)$. For example, it was reported that the miRNA-15a/plasminogen activator inhibitor 2 axis promotes the migration of cholangiocarcinoma cells (9). miR-122 and other miRNAs may have potential as circulating biomarkers in drug-induced liver injury (10). miRNA-351 directly targets the vitamin D receptor to promote schistosomiasis-induced hepatic fibrosis (11). However, to the best of our knowledge, there have been no studies focusing on the miRNA-mRNA network involved in liver fibrosis to date.

In the current study, the expression profiles of miRNAs and mRNAs in liver fibrosis were investigated and compared with normal liver samples. The identification of novel differentially expressed miRNAs may provide novel insights, allowing for the early diagnosis and treatment of liver fibrosis. A total 
of 71 differentially expressed miRNAs (including 56 upregulated and 15 downregulated miRNAs) in were identified fibrotic tissues compared with normal liver tissues. Integrated analysis with human liver cirrhosis data from Gene Expression Omnibus (GEO) datasets revealed 5 miRNAs that were significantly increased in both human and mouse fibrotic liver tissues. A functional miRNA-mRNA network was constructed based on their inverse correlation. Target mRNAs identified in this network were further confirmed in activated hepatic stellate cells (HSCs). The results of the present study may provide novel insights to increase our understanding of the underlying mechanism involved in liver fibrosis.

\section{Materials and methods}

Liver fibrosis model and RNA isolation. A mouse model of liver fibrosis was constructed and RNA was isolated as previously reported (12). In brief, 6-week-old male C57BL/6 mice (weight, $20 \mathrm{~g}$ ) were purchased from Shanghai Laboratory Animal Center (Shanghai, China) and maintained under a $12 \mathrm{~h}$ light/dark cycle with $40-60 \%$ humidity, at $22-25^{\circ} \mathrm{C}$, with free access to food and water. Following acclimatization for one week, the 12 mice were randomly divided into control and treatment groups ( $\mathrm{n}=6 /$ each group). $\mathrm{CCl}_{4}(0.5 \mu \mathrm{l} / \mathrm{g}$ body weight; Sigma-Aldrich; Merck KGaA, Darmstadt, Germany) diluted nine times in corn oil (Sigma-Aldrich; Merck KGaA, Darmstadt, Germany) was administered to mice via intraperitoneal injection by two times per week, for 8 weeks. The control group was injected with an equivalent volume of corn oil. After 8 weeks, mice were anaesthetized by intraperitoneal injection of $0.8 \%$ pentobarbital (Sigma-Aldrich; Merck KGaA, Darmstadt, Germany). and sacrificed and liver tissues were harvested. Total RNA was isolated from four fibrotic and four normal liver tissues using TRIzol reagent (Invitrogen; Thermo Fisher Scientific, Inc., Waltham, MA, USA) according to the manufacturer's instructions. The study was approved by the Ethical Committee of Fudan University and all experiments were performed according to the approved guidelines and regulations.

Histopathology analyses and immunohistochemistry. Liver specimens were fixed in $4 \%$ formaldehyde (Sigma-Aldrich; Merck $\mathrm{KGaA}$ ) at room temperature for $12 \mathrm{~h}$, dehydrated, embedded in paraffin at room temperature for $1 \mathrm{~h}$ and sectioned at $3 \mathrm{um}$. The sections were processed for hematoxylin and eosin (H\&E) and Masson's trichrome (both Sigma-Aldrich; Merck KGaA) staining for assessment of the degree of liver fibrosis. For the H\&E staining, the tissue section was incubated at $65^{\circ} \mathrm{C}$ for $1 \mathrm{~h}$ for dewaxing. Samples were stained with hematoxylin for $1 \mathrm{~min}$, followed by eosin staining for $10 \mathrm{sec}$ at room temperature, and, then the samples were dried at room temperature and sealed. The middle part of the visual field was examined by light microscopy. Masson Trichrome staining was performed following the protocol of trichrome stain (Masson) kit from Sigma-Aldrich. Deparaffinized the slides to deionized water, then mordanted in preheated Bouin's Solution at $56^{\circ} \mathrm{C}$ for $15 \mathrm{~min}$. Cooled the slides in tap water $\left(18-26^{\circ} \mathrm{C}\right)$ and washed in running tap water to remove yellow color from sections. Put the slides in Working Weigert's Iron Haematoxylin Solution for $5 \mathrm{~min}$, Biebrich Scarlet-Acid Fucshin for $5 \mathrm{~min}$, working
Phosphotungstic/Phosphomolybdic Acid Solution for $5 \mathrm{~min}$, Aniline Blue Solution for $5 \mathrm{~min}, 1 \%$ acetic acid for $2 \mathrm{~min}$ both at room temperature. Finally, rinsed slides, dehydrated through alcohol and cleared in xylene. A second set of tissue sections $(0.5 \times 0.5 \mathrm{~cm})$ were prepared for immunohistochemical assessment of the tissues. Briefly, endogenous peroxidase activity was blocked by immersion of deparaffinized sections in $3 \%$ $\mathrm{H} 2 \mathrm{O} 2$ in methanol for $30 \mathrm{~min}$ at room temperature. Antigen retrieval was performed by steaming slides in $0.01 \mathrm{M}$ citrate buffer $(\mathrm{pH}$ 6.0) and heated them until the temperature reached $95-100^{\circ} \mathrm{C}$ for $30 \mathrm{~min}$. The samples were incubated for $30 \mathrm{~min}$ at room temperature in 5\% normal blocking serum, and incubated with a 1:100 dilution of polyclonal rabbit anti- $\alpha$-SMA (1:200, cat. no. ab5694, Abcam, Cambridge, UK) overnight at $4^{\circ} \mathrm{C}$. The slides were then incubated with HRP-conjugated goat anti-rabbit secondary antibody (1:2,000, ab7090; Abcam) for $60 \mathrm{~min}$ at room temperature. Between each incubation, sections were washed three times with PBS. Sections were developed with 3,3-diaminobenzidine tetrahydrochloride and hydrogen peroxide and subsequently counterstained with hematoxylin. The sections were imaged using a Nikon Eclipse 80i microscope (Nikon Corporation, Tokyo, Japan). Hematoxylin and eosin, $\alpha$-smooth muscle actin (SMA) and Masson staining demonstrated that liver fibrosis was successfully established, which was also verified in our previous study (12).

miRNA sequencing. Total RNA was used to prepare miRNA libraries and the purified libraries were sequenced on an Illumina HiSeq 2000 platform (Illumina, Inc., San Diego, CA, USA). In brief, the raw data were processed with R software (version 3.3.2; www.r-project.org) to ensure its quality. Clean data were mapped to the Ensemble database (GRCh37) (13) and compared with miRBase (release 21; www.mirbase.org) to identify mature miRNAs. The count and reads per million total read values of the miRNAs were collected for each sample. Individual miRNAs were analyzed to identify significant differences using the DESeq2 package (version 1.4.0) (14) in $\mathrm{R}$. The parameters for differentially expressed miRNAs were set with a false discovery rate of $<0.05$ and $\log 2$ fold change (FC) $\mid>=1$. The differentially expressed miRNAs are shown by heatmap and volcano plot using $\mathrm{R}$ software The miRNA expression profiles (GSE49012 and GSE40744) in human liver cirrhosis tissues were downloaded from publicly available Gene Expression Omnibus (GEO) datasets (www.ncbi.nlm. nih.gov/geo) $(15,16)$.

miRNA target prediction. miRWalk (version 2.0) (17) collects 13 predicted data sets from existing miRNA target databases to predict all possible miRNA-mRNA interactions. Putative interactions between the sequenced miRNAs and mRNAs were evaluated using miRWalk. The search was restricted to miRNAs with a minimum seed length of seven nucleotides and binding sites in the 3'untranslated region (UTR) of target genes. $\mathrm{P}<0.05$ was considered to indicate a statistically significant difference.

Functional analysis. To explore the functional roles of target genes, the Database for Annotation, Visualization and Integrated Discovery (version 6.8; www.david.ncifcrf. gov) software $(18,19)$ was used, which integrates the Gene 
Table I. Primers used for RT-qPCR.

\begin{tabular}{|c|c|c|}
\hline Gene (human) & Forward primer ( $5^{\prime}$ to $\left.3^{\prime}\right)$ & Reverse primer (5'to $\left.3^{\prime}\right)$ \\
\hline RELB & CAGCCTCGTGGGGAAAGAC & GCCCAGGTTGTTAAAACTGTGC \\
\hline RAP1A & CGTGAGTACAAGCTAGTGGTCC & CCAGGATTTCGAGCATACACTG \\
\hline PPP3CB & CCCCAACACATCGCTTGACAT & GGCAGCACCCTCATTGATAATTC \\
\hline ARRB1 & AAAGGGACCCGAGTGTTCAAG & CGTCACATAGACTCTCCGCT \\
\hline MAP2K4 & TGCAGGGTAAACGCAAAGCA & CTCCTGTAGGATTGGGATTCAGA \\
\hline FGF1 & CTCCCGAAGGATTAAACGACG & GTCAGTGCTGCCTGAATGCT \\
\hline MAP3K4 & CTCGACAGATGAAACGCATGT & CCAGTGTCTTTATGTGGAGGC \\
\hline PPKCB & AGCCCCACGTTTTGTGACC & GCTGGGAACATTCATCACGC \\
\hline GAPDH & ACAACTTTGGTATCGTGGAAGG & GCCATCACGCCACAGTTTC \\
\hline \multicolumn{3}{|l|}{ Gene (mouse) } \\
\hline Relb & CACCGGGTACACCCACATAG & ATGCCCAGGTTGTTAAAGCTG \\
\hline Rap1a & ATGCGTGAGTACAAGCTAGTAGT & AАTCTACCTCGACTTGCTTTCTG \\
\hline Ppp3cb & AAAGCGTGCTGACACTCAAG & TGGAGAGAATCCTCGTATTGCT \\
\hline Arrb1 & AGGCAAGCCCCAATGGAAAG & AGTGTCACGTAGACTCGCCTT \\
\hline Map2k4 & AATCGACAGCACGGTTTACTC & GCAGTGAAATCCCAGTGTTGTT \\
\hline Fgf1 & GGGGAGATCACAACCTTCGC & GTCCCTTGTCCCATCCACG \\
\hline Map3k4 & GAGTCGGCTCGCAAAAGTATG & GTGAGGTGCCGTAGAGAGTC \\
\hline Ppkcb & ATGAGTTCGTCACGTTCTCCT & CCATACAGCAGCGATCCACAG \\
\hline Gapdh & AGGTCGGTGTGAACGGATTTG & GGGGTCGTTGATGGCAACA \\
\hline
\end{tabular}

Ontology (GO) and Kyoto Encyclopedia of Genes and Genomes (KEGG) databases to analyze biological functions. Fisher's exact test was performed to evaluate the enrichment values of GO terms and KEGG pathways. $\mathrm{P}<0.05$ was considered to indicate a statistically significant difference. The miRNA-mRNA interaction regulatory network was constructed using Cytoscape software (20) (version 3.3.0; www.cytoscape.org).

Activation of LX-2 cells. LX-2 HSC cells were cultured in DMEM (Invitrogen; Thermo Fisher Scientific, Inc.) supplemented with $10 \%$ fetal bovine serum (FBS, Invitrogen; Thermo Fisher Scientific, Inc.) in a humidified atmosphere containing $5 \% \mathrm{CO}_{2}$ at $37^{\circ} \mathrm{C}$ for $24 \mathrm{~h}$. LX-2 cells were starved for $12 \mathrm{~h}$ in serum-free DMEM, following which they were treated with 10 ng/ml TGF- $\beta$ (R\&D Systems China Co., Ltd., Shanghai, China). After 24 h of TGF- $\beta$ treatment, the cells were harvested for RNA isolation and reverse transcription-quantitative polymerase chain reaction (RT-qPCR).

$R T$-qPCR. SYBR Green-based qPCR (Thermo Fisher Scientific, Inc.) was used to measure mRNA expression. Total RNA $(2 \mu \mathrm{g})$ was reverse transcribed into cDNA using the reverse transcription kit (Takara Biotechnology Co., Ltd. Dalian, China). The protocol for reverse transcription was as follows: $25^{\circ} \mathrm{C}$ for $10 \mathrm{~min} ; 37^{\circ} \mathrm{C}$ for $120 \mathrm{~min}$ and $85^{\circ} \mathrm{C}$ for $5 \mathrm{~min}$. The thermocycling conditions for RT-qPCR were as follows: Initial denaturation at $95^{\circ} \mathrm{C}$ for $10 \mathrm{~min}$; 40 cycles of denaturation at $95^{\circ} \mathrm{C}$ for $30 \mathrm{sec}$, annealing at $55^{\circ} \mathrm{C}$ for $30 \mathrm{sec}$ and extension at $72^{\circ} \mathrm{C}$ for $30 \mathrm{sec}$; followed by melting curve analysis. All experiments were performed in triplicate. The comparative $\Delta \mathrm{Cq}$ method (21) was used to analyze the data, with GAPDH as an endogenous reference gene. The RT-PCR primers are listed in Table I.

Western blotting. Protein samples were extracted from cells using radioimmunoprecipitation lysis buffer (Beyotime Biotechnology, Inc., Haimen, China). Total protein concentration was quantified by Bicinchoninic acid Protein Assay kit (Beyotime Biotechnology). Equal amounts of protein (30 $\mu \mathrm{g} /$ lane) were separated on $8-12 \%$ polyacrylamide gels and transferred onto polyvinylidene difluoride membranes. Subsequently, the membranes were blocked with 5\% fat-free dry milk at room temperature for $1 \mathrm{~h}$. Membranes were incubated with mouse anti- $\alpha$-SMA (cat. no. A5228, 1:1,000; Sigma-Aldrich; Merck KGaA), anti-collagen $\alpha-1(\mathrm{I})$ chain (COL1 $\alpha 1$; cat. no. ABT123, 1:1,000, EMD Millipore, Billerica, MA, USA) or anti-GAPDH (cat. no. ab8245, 1:4,000; Abcam, Cambridge, UK) antibodies in Tris buffered saline with $0.1 \%$ Tween-20 (TBST) overnight on a shaker at $4^{\circ} \mathrm{C}$. Membranes were subsequently washed three times in TBST and incubated with horseradish peroxidase (HRP)-conjugated rabbit anti-mouse IgG secondary antibodies (1:5,000, cat. no. ab6728, Abcam) at room temperature for $1 \mathrm{~h}$. Immunoblots were visualized using Enhanced Chemiluminescent Plus western blotting substrate (Thermo Fisher Scientific, Inc.).

Statistical analysis. Student's t-test was performed to compare two variables using SPSS v20 software (IBM Corp., Armonk, NY, USA). All data are expressed as the mean \pm standard deviation. $\mathrm{FC} \geq 2$ and $\mathrm{P}<0.05$ were considered to indicate a statistically significant difference. All experiments were repeated in triplicate. 
A

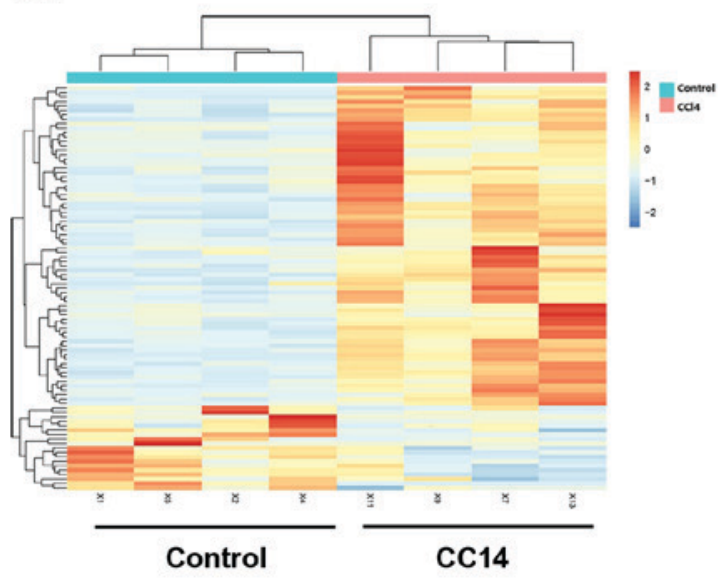

C

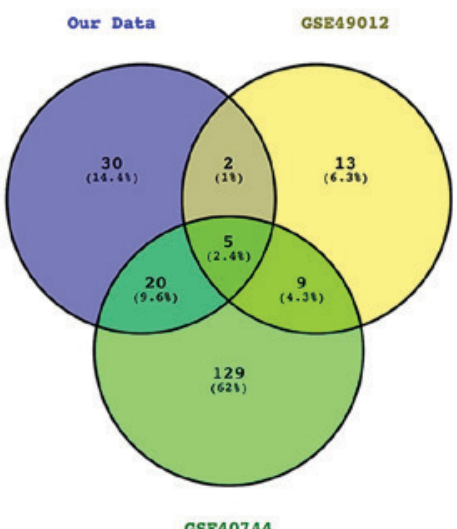

B

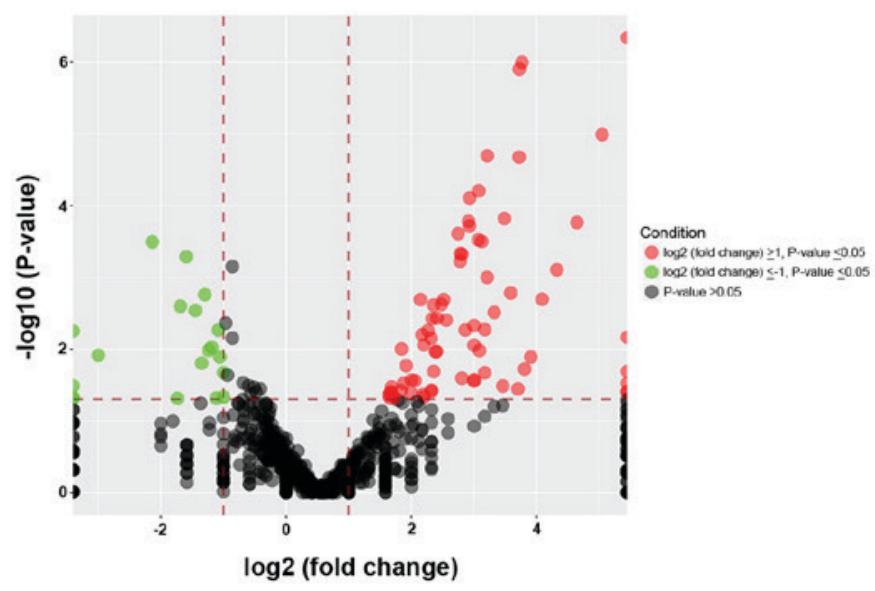

D

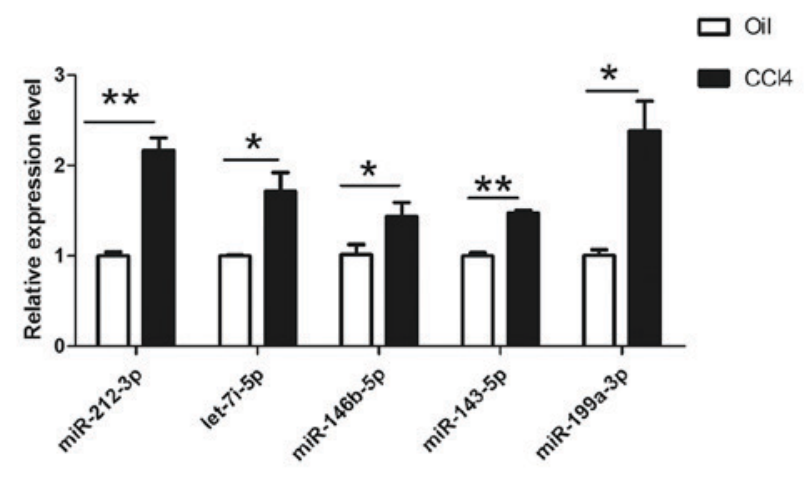

Figure 1. Expression profiles of miRNAs in liver fibrosis. Red indicates high expression and green indicates low expression. (A) Heatmap plot of miRNAs. (B) Volcano plot of dysregulated miRNAs. (C) Venn diagram showing the number of miRNAs that were dysregulated between the miRNA profile from the present study and clinical liver fibrosis miRNA profiles from GSE49012 and GSE40744 datasets. (D) Quantitative polymerase chain reaction results validating the miRNA sequencing results. ${ }^{*} \mathrm{P}<0.05,{ }^{* *} \mathrm{P}<0.01$. miRNA, microRNA.

\section{Results}

Identification of differentially expressed miRNAs in liver fibrosis. To identify miRNAs that may be significant in liver fibrogenesis, miRNA sequencing was performed to compare their expression between fibrotic liver and normal liver tissues. As shown in Fig. 1, 71 miRNAs that were significantly differentially expressed between fibrotic liver and normal liver tissues were identified. Among these, 56 were upregulated and 15 were downregulated in fibrotic samples. A heatmap and volcano plot were produced to show the differentially expressed miRNAs using R software, of which the top 20 dysregulated miRNAs were summarized and listed in Table II. To identify conserved miRNAs in human clinical samples and animal experiments, two miRNA profiles (GSE49012 and GSE40744) were downloaded from publicly available GEO datasets (www.ncbi.nlm.nih.gov/geo). The data suggested that five miRNAs (miR-212-3p, miR-146b-5p, let-7i-5p, miR-143-5p and miR-199a-3p) were also upregulated in human liver fibrotic samples, which was consistent with the results of the present study (Fig. 1C). RT-qPCR was performed to verify the expression of these five upregulated miRNAs in $\mathrm{CCl}_{4}$-induced fibrotic and control normal liver tissues. It was confirmed that miR-212-3p, miR-146b-5p, let-7i-5p, miR-143-5p and miR-199a-3p were significantly increased in $\mathrm{CCl}_{4}$-treated liver tissues compared with control samples (Fig. 1D).

GO analysis and pathway analysis. Bioinformatics analyses were used to analyze miRNA function based on their target mRNAs. GO analysis was performed to evaluate mRNA enrichments in terms of biological process, cellular component and molecular function. The top 10 enriched GO terms were involved in the regulation of cellular and metabolic processes (Fig. 2A). KEGG analysis revealed the top 10 pathways, which included the mitogen-activated protein kinase (MAPK), phosphoinositide 3-kinase (PI3K)-protein kinase B (Akt), focal adhesion and Wnt signaling pathways. All of these pathways are associated with HSC activation and extracellular matrix ECM remodeling (22-24). These results suggested that some differentially expressed miRNAs may be involved in the progression of liver fibrosis (Fig. 2B).

Construction of the miRNA-mRNA interaction network. To further investigate the association between distinct 
Table II. Dysregulated microRNAs in $\mathrm{CCl}_{4}$-induced liver fibrosis $(>2$-fold; $\mathrm{P}<0.05)$.

A, Upregulated

\begin{tabular}{lccr}
\hline & \multicolumn{2}{c}{$\mathrm{CCl}_{4} /$ Corn Oil } & \multicolumn{2}{c}{$\mathrm{CCl}_{4} /$ Corn Oil } \\
\cline { 2 - 3 } Probe ID & $\mathrm{Log}_{2}$ change & Fold-change & P-value \\
\hline mmu-miR-212-3p & 5.04 & 33.0 & 0.04778 \\
mmu-miR-3081-3p & 4.64 & 25.0 & 0.03257 \\
mmu-miR-1983 & 4.32 & 20.0 & 0.02042 \\
mmu-miR-411-3p & 4.09 & 17.0 & 0.03905 \\
mmu-miR-147-5p & 3.91 & 15.0 & 0.03943 \\
mmu-miR-543-3p & 3.81 & 14.0 & 0.02985 \\
mmu-miR-582-3p & 3.77 & 13.6 & 0.00001 \\
mmu-miR-708-3p & 3.72 & 13.2 & 0.00017 \\
mmu-miR-134-5p & 3.72 & 13.2 & 0.00078 \\
mmu-miR-376b-3p & 3.70 & 13.0 & 0.00202 \\
\hline
\end{tabular}

B, Downregulated

\begin{tabular}{|c|c|c|c|}
\hline \multirow[b]{2}{*}{ Probe ID } & \multirow{2}{*}{$\frac{\mathrm{CCl}_{4} / \text { Corn Oil }}{\log _{2} \text { change }}$} & \multirow{2}{*}{$\frac{\mathrm{CCl}_{4} / \text { Corn Oil }}{\text { Fold-change }}$} & \multirow[b]{2}{*}{ P-value } \\
\hline & & & \\
\hline mmu-miR-193a-5p & -1 & 0.50 & 0.02130 \\
\hline mmu-miR-26b-3p & -1 & 0.50 & 0.04648 \\
\hline mmu-miR-137-3p & -1.12 & 0.46 & 0.04818 \\
\hline mmu-miR-871-3 & -1.19 & 0.44 & 0.00943 \\
\hline mmu-miR-1948-5p & -1.23 & 0.43 & 0.01038 \\
\hline mmu-miR-365-3p & -1.30 & 0.41 & 0.00174 \\
\hline mmu-miR-192-3p & -1.35 & 0.39 & 0.01571 \\
\hline mmu-miR-741-3p & -1.45 & 0.37 & 0.00290 \\
\hline mmu-miR-6390 & -1.60 & 0.33 & 0.00052 \\
\hline mmu-miR-122 & -1.69 & 0.31 & 0.00252 \\
\hline
\end{tabular}

miRNAs and target mRNAs in fibrotic liver tissues, the mRNA sequencing profile of the $\mathrm{CCl}_{4}$-induced liver fibrosis mouse model was extracted from our previous study (12). A miRNA-mRNA regulatory network associated with liver fibrosis was constructed based on the inverse expression relationships between the five miRNAs upregulated in both mouse and human fibrotic liver tissues and mRNAs significantly enriched in the MAPK, PI3K-Akt, focal adhesion and Wnt signaling pathways. As shown in Fig. 3A, the network contained five miRNAs, 22 mRNAs and 23 miRNA-mRNA connections. Target mRNAs in the network, such as RELB proto-oncogene (RELB), RAS-related protein 1a (RAP1A), protein phosphatase 3 catalytic subunit $\beta$ (PPP3CB), arrestin $\beta 1$ (ARRB1), fibroblast growth factor 1 (FGF1), protein kinase $\mathrm{C} \beta$ (PRKCB) and members of the MAPK family (MAP3K4, MAPK8, MAPKAPK5, MAP2K5, MAP2K4, MAP3K1), were summarized in Table III. The RT-qPCR results revealed that the expression of RELB, RAP1A, PPP3CB, MAP2K4, ARRB1, MAP3K4, FGF1 and $\mathrm{PRKCB}$ was decreased in $\mathrm{CCl}_{4}$-induced fibrotic liver tissues
(Fig. 3B). The integrated analysis revealed their regulatory interactions in liver fibrosis.

Target mRNAs in the network are associated with HSC activation. HSCs have an essential role in liver fibrosis, in which they undergo activation or transdifferentiation from quiescent to myofibroblast-like cells as a result of injury (25). LX-2 is a widely used human HSC line that can be activated by TGF- $\beta 1$. To further target mRNAs in HSC activation, the expression of mRNAs in LX-2 cells was examined after $24 \mathrm{~h}$ of TGF- $\beta 1(10 \mathrm{ng} / \mathrm{ml})$ stimulation. Following stimulation, the expression of COL $1 \alpha 1$ and $\alpha$-SMA was significantly upregulated (Fig. 4A), suggesting that LX-2 cells were activated. In addition, miR-212-3p, miR-146b-5p, let-7i-5p, miR-143-5p and $\mathrm{miR}-199 \mathrm{a}-3 \mathrm{p}$ expression was significantly upregulated in LX-2 cells following TGF- $\beta 1$ treatment (Fig. 4B). Of the 22 mRNAs assessed, the expression of RELB, RAP1A, PPP3CB, MAP2K4, ARRB1, MAP3K4, FGF1 and PRKCB was also significantly decreased (Fig. 4C). The expression pattern of these mRNAs was inverse to that of their regulatory 
A

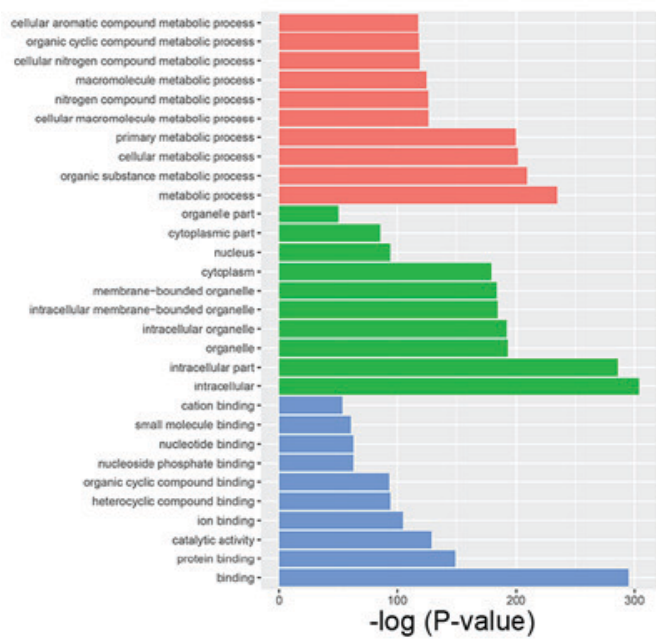

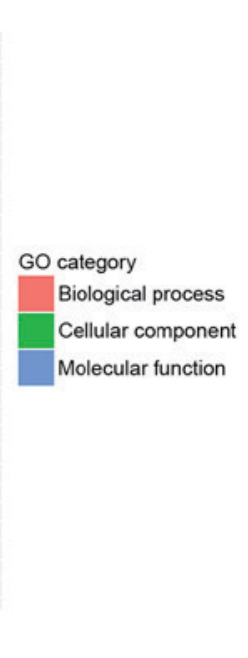

B KEGG pathways

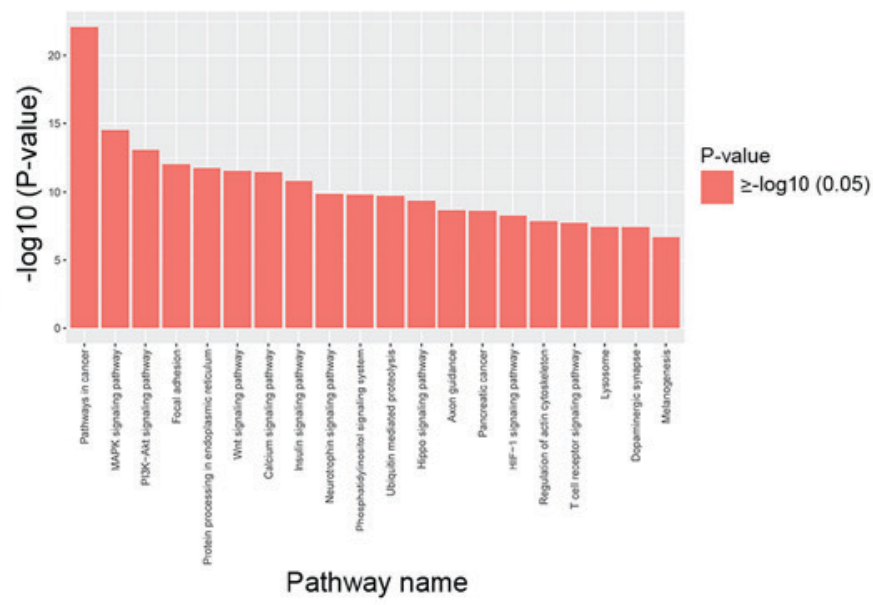

Figure 2. GO enrichment and KEGG pathways of predicted miRNA target genes. (A) GO enrichment results showing differences in target genes based on their distribution by GO terms. (B) The y-axis represents pathway enrichment and the x-axis represents KEGG pathways. GO, gene ontology; KEGG, Kyoto Encyclopedia of Genes and Genomes.

A

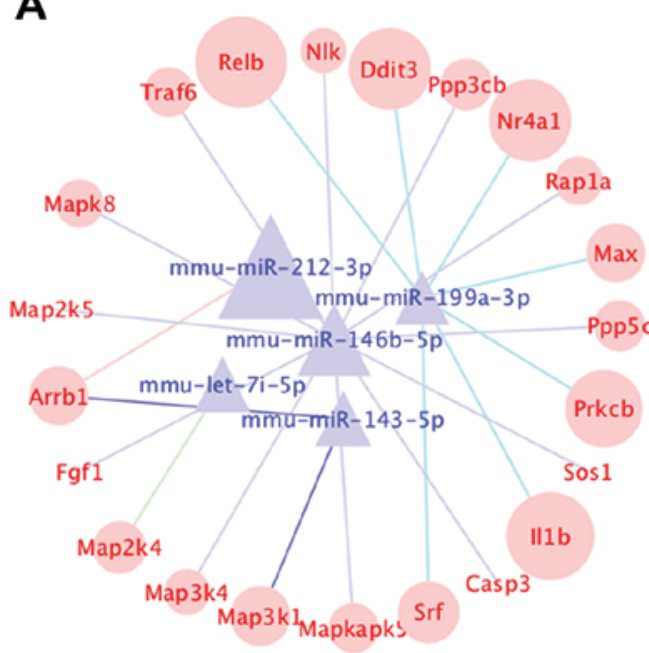

B

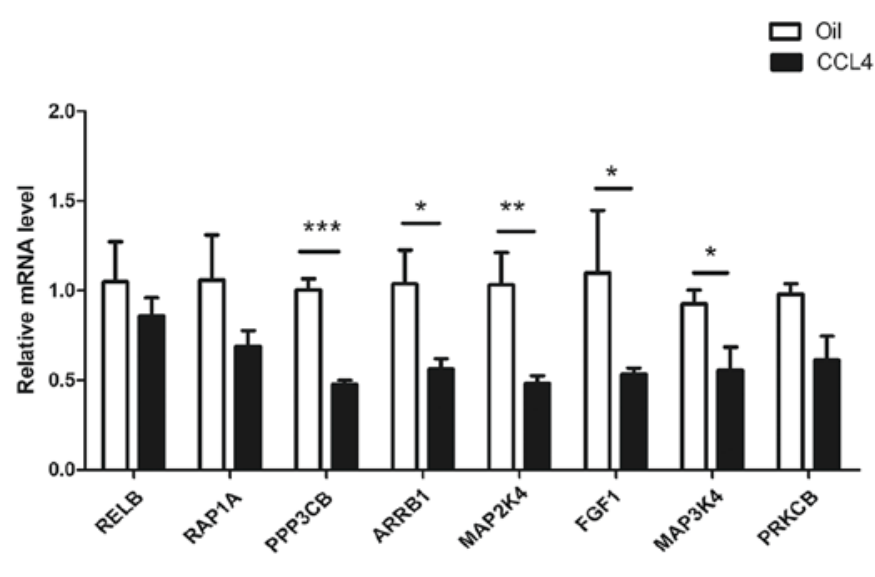

Figure 3. Construction of a miRNA-mRNA co-expression network. (A) The co-expression network was comprised of five highly conserved miRNAs and 22 mRNAs. The different color lines represent different miRNAs. (B) mRNA expression of RELB, RAP1A, PPP3CB, ARRB1, MAP2K4, FGF1, MAP3K4 and PRKCB in $\mathrm{CCl}_{4}$-induced fibrotic and normal liver tissues. ${ }^{*} \mathrm{P}<0.05,{ }^{* * *} \mathrm{P}<0.01,{ }^{* * * *} \mathrm{P}<0.001$. Relb, RELB proto-oncogene; RAP1A, RAS-related protein 1a; PPP3CB, protein phosphatase 3 catalytic subunit $\beta$; ARRB1, arrestin $\beta 1$; MAP2/3K, mitogen-activated protein kinase kinase; FGF1, fibroblast growth factor 1 ; PRKCB, protein kinase $\mathrm{C} \beta$.

miRNAs (miR-199a-3p for RELB and PRKCB; miR-146b-5p for RAP1A, FGF1 and PPP3CB; miR-143a-3p for MAP3K4; and let-7i-5p for MAP2K4) in the activated HSCs. These results suggested that these miRNAs have an inhibitory effect on their target genes and may serve important roles in liver fibrosis.

\section{Discussion}

An increasing number of studies have reported that miRNAs serve a role in regulating the progression and metastasis of various cancers (26-30). However, the role of miRNAs and their specific mechanism of action in liver fibrogenesis remain to be elucidated (31). In the present study, miRNA sequencing was performed in $\mathrm{CCl}_{4}$-induced liver fibrosis and integrated with miRNA array data from clinical liver fibrosis samples to compare miRNA expression profiles between fibrotic and normal liver tissues. It was demonstrated that five miRNAs (miR-212-3p, miR-146b-5p, let-7i-5p, miR-199a-3p and miR-143-5p) were significantly upregulated in $\mathrm{CCl}_{4}$-treated livers and human liver cirrhosis samples compared with normal liver tissues. Furthermore, GO and KEGG pathway enrichment analysis was performed for miRNA target genes to identify the signaling pathways involved in hepatic fibrosis. GO enrichment analysis indicated that the majority of the miRNA target genes were involved in cellular metabolic processes closely associated with the onset and development of hepatic fibrosis 
Table III. Putative target genes involved in the microRNA-mRNA network.

\begin{tabular}{|c|c|c|c|}
\hline Gene & Official full name & Reference sequence & Targeting miRNA \\
\hline ARRB1 & Arrestin $\beta 1$ & NM_178220.3 & $\begin{array}{l}\text { mmu-miR-212-3p } \\
\text { mmu-miR-143-5p }\end{array}$ \\
\hline NLK & Nemo like kinase & NM_008702.3 & mmu-miR-146b-5p \\
\hline PPP3CB & $\begin{array}{l}\text { Protein phosphatase } 3 \\
\text { catalytic subunit } \beta\end{array}$ & NM_008914.3 & mmu-miR-146b-5p \\
\hline MAPK8 & $\begin{array}{l}\text { Mitogen-activated } \\
\text { protein kinase } 8\end{array}$ & NM_001310454.1 & mmu-miR-146b-5p \\
\hline MAP3K4 & $\begin{array}{l}\text { Mitogen-activated } \\
\text { protein kinase kinase } \\
\text { kinase } 4\end{array}$ & NM_011948.2 & mmu-miR-146b-5p \\
\hline SOS1 & $\begin{array}{l}\text { SOS Ras/Rac guanine } \\
\text { nucleotide exchange } \\
\text { factor } 1\end{array}$ & NM_009231.2 & mmu-miR-146b-5p \\
\hline FGF1 & Fibroblast growth factor 1 & NM_001033789.2 & mmu-miR-146b-5p \\
\hline TRAF6 & $\begin{array}{l}\text { TNF receptor-associated } \\
\text { factor } 6\end{array}$ & NM_009424.3 & mmu-miR-146b-5p \\
\hline RAP1A & RAS-related protein 1a & NM_145541.5 & mmu-miR-146b-5p \\
\hline MAPKAPK5 & $\begin{array}{l}\text { MAP kinase-activated } \\
\text { protein kinase } 5\end{array}$ & NM_010765.2 & mmu-miR-146b-5p \\
\hline PPP5 & $\begin{array}{l}\text { Protein phosphatase } 5, \\
\text { catalytic subunit }\end{array}$ & NM_011155.2 & mmu-miR-146b-5p \\
\hline CASP3 & Caspase 3 & NM_001284409.1 & mmu-miR-146b-5p \\
\hline MAP2K & $\begin{array}{l}\text { mitogen-activated } \\
\text { protein kinase kinase } 5\end{array}$ & NM_011840.2 & mmu-miR-146b-5p \\
\hline MAP2K4 & $\begin{array}{l}\text { Mitogen-activated } \\
\text { protein kinase kinase } 4\end{array}$ & NM_001316368.1 & mmu-let-7i-5p \\
\hline DDIT & $\begin{array}{l}\text { DNA-damage inducible } \\
\text { transcript } 3\end{array}$ & NM_001003913.2 & mmu-miR-199a-3p \\
\hline MAX & Max protein & NM_008558.2 & mmu-miR-199a-3p \\
\hline NR4A1 & $\begin{array}{l}\text { Nuclear receptor } \\
\text { subfamily } 4\end{array}$ & NM_010444.2 & mmu-miR-199a-3p \\
\hline SRF & Serum response factor & NM_020493.2 & mmu-miR-199a-3p \\
\hline IL1B & Interleukin $1 \beta$ & NM_008361.4 & mmu-miR-199a-3p \\
\hline PRKCB & Protein kinase $C \beta$ & NM_008855.2 & mmu-miR-199a-3p \\
\hline RELB & $\begin{array}{l}\text { Reticuloendotheliosis } \\
\text { viral (v-rel) oncogene related B }\end{array}$ & NM_009046.2 & mmu-miR-199a-3p \\
\hline MAP3K1 & $\begin{array}{l}\text { Mitogen-activated } \\
\text { protein kinase kinase kinase } 1\end{array}$ & NM_011945.2 & mmu-miR-143-5p \\
\hline
\end{tabular}

The results of KEGG pathway enrichment analysis indicated a total of 159 signaling pathways that may be essential for the development of liver fibrosis. Several of these pathways were already known to be involved in the progression of liver fibrosis, such as the MAPK, focal adhesion, Wnt, p53, mTOR and PI3K-Akt signaling pathways (22-24). Various metabolic pathways were also identified in the present study's analysis such as glycan metabolism, cysteine and methionine metabolism and tyrosine metabolism pathway.

HSCs are an important cell type in the process of liver fibrosis, as they can be activated by a large number of cytokines secreted by damaged hepatocytes in chronic liver injury.
Activated HSCs promote the deposition of ECM, which in turn leads to the development of liver fibrosis. (32). Therefore, several molecules involved in the activation of HSCs may have potential as therapeutic targets in liver fibrosis (33-35). Previous studies have demonstrated that MAPK signaling plays a key role in HSC activation and liver fibrogenesis: MAPK signaling promotes the activation and proliferation of HSCs, increases collagen mRNA stability and leads to the accumulation of ECM $(36,37)$. These findings suggest that dysregulated miRNAs that affect MAPK signaling may affect HSC activation in hepatic fibrosis. In the current study, MAPK-related genes, including RELB, RAP1A, PPP3CB, 


\section{Control TGF- $\beta$}

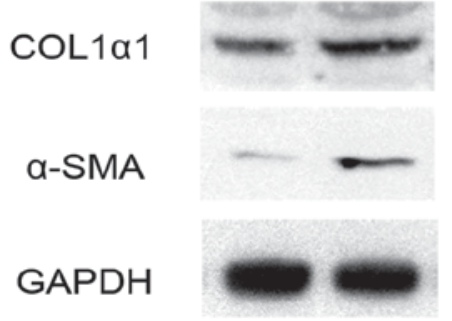

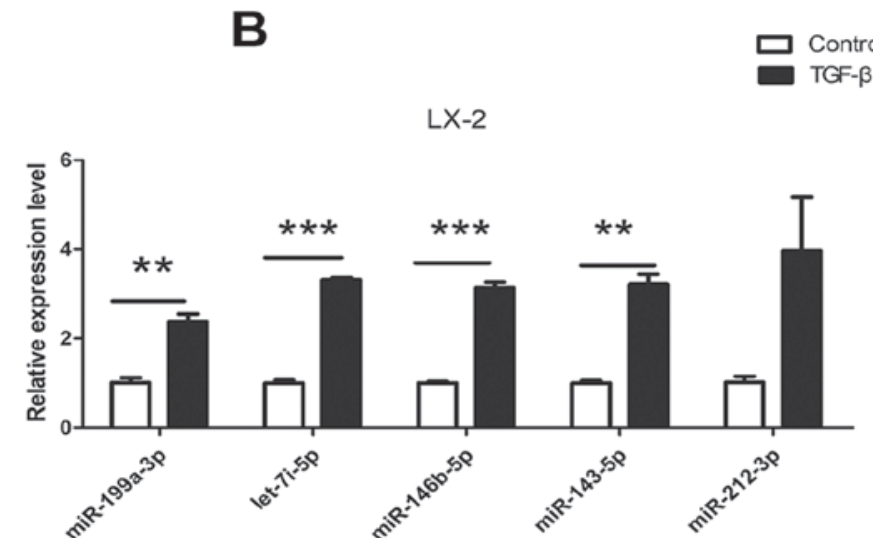

$L X-2$

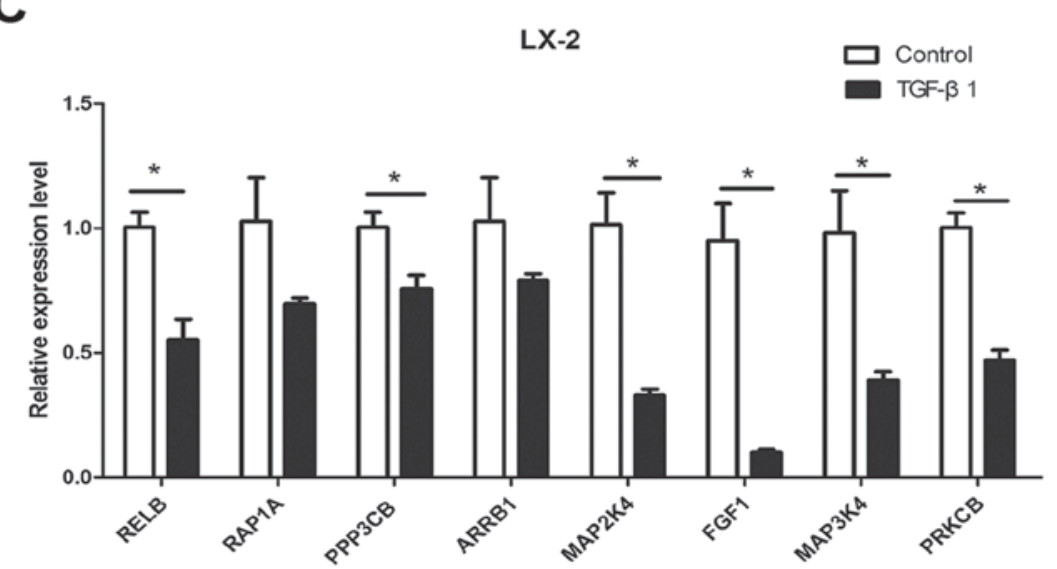

Figure 4. Expression of dysregulated miRNAs and target mRNAs in LX-2 cells following TGF- $\beta$ stimulation for 24 h. (A) $\alpha$-SMA and COL1 $\alpha 1$ protein expression in LX-2 cells following $10 \mathrm{ng} / \mathrm{ml}$ TGF- $\beta$ stimulation. (B) RT-qPCR results for miR-212-3p, miR-146b-5p, let-7i-5p, miR-143-5p and miR-199a-3p. (C) RT-qPCR results for RELB, RAP1A, PPP3CB, ARRB1, MAP2K4, FGF1, MAP3K4 and PRKCB. " $\mathrm{P}<0.05,{ }^{* * *} \mathrm{P}<0.01,{ }^{* * * *} \mathrm{P}<0.001 . \alpha$-SMA, $\alpha$-smooth muscle actin; COL1 $\alpha 1$, collagen $\alpha-1(\mathrm{I})$ chain; TGF- $\beta$, transforming growth factor- $\beta$; miR, microRNA; RELB, RELB proto-oncogene; RAP1A, RAS-related protein 1a; PPP3CB, protein phosphatase 3 catalytic subunit $\beta$; ARRB1, arrestin $\beta$ 1; MAP2/3K, mitogen-activated protein kinase kinase; FGF1, fibroblast growth factor 1; PRKCB, protein kinase $\mathrm{C} \beta$.

ARRB1, MAP2K4, FGF1, MAP3K4 and PRKCB, were dysregulated in TGF- $\beta$-induced activation of HSCs, suggesting that they may be essential for HSC activation.

A number of studies have reported that miRNAs are involved in the regulation of liver fibrosis (38-40). let-7i, miR-342-3p, miR-188-5p and miR-34a are upregulated in $\mathrm{CCl}_{4}$-treated fibrotic livers compared with control samples, while miR-202, miR-378b and miR-378d are downregulated (41). It was previously reported that a module containing five downregulated miRNAs (miR-130b-3p, miR-148a-3p, miR-345-5p, miR-378a-3p and miR-422a) was associated with HSC activation (42). The miR-199 family is closely associated with the progression of liver fibrosis in humans and mice (43). In our previous study, it was demonstrated that the miR-212 promotes HSC activation by targeting Smad family member 7 (44). Similarly, the results of the present study revealed that miR-342-3p, let-7i, miR-212 and miR-199 were significantly upregulated in cirrhotic liver tissues, whereas miR-378a-3p was significantly downregulated. These miRNAs may therefore be promising biomarkers for the early diagnosis of liver cirrhosis.

In conclusion, the miRNA expression profile generated in the present study revealed that the expression of several miRNAs (miR-212-3p, miR-146b-5p, let-7i-5p,
miR-143-5p, miR-199a-3p) was associated with liver fibrosis. A miRNA-mRNA network was identified, comprised of 5 miRNAs and 22 mRNAs that were associated with liver fibrosis. This study may provide novel insights and potential biomarkers for the early diagnosis and treatment of liver fibrosis. Future studies should focus on the biological functions of these miRNAs.

\section{Acknowledgements}

Not applicable.

\section{Funding}

This study was supported by grants from the National Natural Science Foundation of China (grant nos. 81420108005 and 81300327) and the Ministry of Science and Technology of China (grant no. 2013CB945401).

\section{Availability of data and materials}

The analyzed data sets generated during the study are available from the corresponding author on reasonable request. 


\section{Authors' contributions}

YZ and JinL performed the cell experiments, analyzed the data and wrote the paper; YM, JW and JieZ conceived the mouse experimental design and performed the animal experiments. JieL and JunZ designed the study and revised the paper.

\section{Ethics approval and consent to participate}

The animal study was approved by the Animal Care and Use Committee at the Fudan University. Animals were anesthetized and sacrificed using acceptable methods.

\section{Patient consent for publication}

Not applicable.

\section{Competing interests}

The authors declare they have no competing interests.

\section{References}

1. Bataller R and Brenner DA: Liver fibrosis. J Clin Invest 115: 209-218, 2005

2. Friedman SL: Liver fibrosis-from bench to bedside. J Hepatol 1 (Suppl 38): S38-S53, 2003.

3. Moreira RK: Hepatic stellate cells and liver fibrosis. Arch Pathol Lab Med 131: 1728-1734, 2007.

4. Ellis EL and Mann DA: Clinical evidence for the regression of liver fibrosis. J Hepatol 56: 1171-1180, 2012.

5. Chen W and Qin C: General hallmarks of microRNAs in brain evolution and development. RNA Biol 12: 701-708, 2015.

6. Grimson A, Farh KK, Johnston WK, Garrett-Engele P, Lim LP and Bartel DP: MicroRNA targeting specificity in mammals: Determinants beyond seed pairing. Mol Cell 27: 91-105, 2007.

7. Wang XW, Heegaard NH and Orum H: MicroRNAs in liver disease. Gastroenterology 142: 1431-1443, 2012.

8. Szabo G and Bala S: MicroRNAs in liver disease. Nat Rev Gastroenterol Hepatol 10: 542-552, 2013.

9. Utaijaratrasmi $\mathrm{P}$, Vaeteewoottacharn $\mathrm{K}$, Tsunematsu $\mathrm{T}$, Jamjantra P, Wongkham S, Pairojkul C, Khuntikeo N, Ishimaru N, Sirivatanauksorn Y, Pongpaibul A, et al: The microRNA-15a-PAI-2 axis in cholangiocarcinoma-associated fibroblasts promotes migration of cancer cells. Mol Cancer 17: $10,2018$.

10. Howell LS, Ireland L, Park BK and Goldring CE: MiR-122 and other microRNAs as potential circulating biomarkers of drug-induced liver injury. Expert Rev Mol Diagn 18: 47-54, 2018.

11. He X, Sun Y, Lei N, Fan X, Zhang C, Wang Y, Zheng K, Zhang D and Pan W: MicroRNA-351 promotes schistosomiasis-induced hepatic fibrosis by targeting the vitamin D receptor. Proc Natl Acad Sci USA 115: 180-185, 2018.

12. Zhang Y, Liu J, Ma Y, Wang J,Zhu J, Liu J and Zhang J: Integrated profiling of long non-coding RNAs and mRNAs identifies novel regulators associated with liver fibrosis. Pathol Res Pract 214 1794-1803, 2018

13. Aken BL, Achuthan P, Akanni W, Amode MR, Bernsdorff F Bhai J, Billis K, Carvalho-Silva D, Cummins C, Clapham P, et al: Ensembl 2017. Nucleic Acids Res 45: D635-d642, 2017.

14. Love MI, Huber W and Anders S: Moderated estimation of fold change and dispersion for RNA-seq data with DESeq2. Genome Biol 15: 550, 2014

15. Vuppalanchi R, Liang T, Goswami CP, Nalamasu R, Li L, Jones D, Wei R, Liu W, Sarasani V, Janga SC and Chalasani N: Relationship between differential hepatic microRNA expression and decreased hepatic cy tochrome P450 3A activity in cirrhosis. Plos One 8: e74471,2013.

16. Diaz G, Melis M, Tice A, Kleiner DE, Mishra L, Zamboni F and Farci P: Identification of microRNAs specifically expressed in hepatitis $\mathrm{C}$ virus-associated hepatocellular carcinoma. Int J Cancer 133: 816-824, 2013.
17. Dweep H and Gretz N: miRWalk2.0: A comprehensive atlas of microRNA-target interactions. Nat Methods 12: 697, 2015.

18. Huang da W, Sherman BT and Lempicki RA: Systematic and integrative analysis of large gene lists using DAVID bioinformatics resources. Nat Protoc 4: 44-57, 2009.

19. Huang da W, Sherman BT and Lempicki RA: Bioinformatics enrichment tools: Paths toward the comprehensive functional analysis of large gene lists. Nucleic Acids Res 37: 1-13, 2009.

20. Shannon P, Markiel A, Ozier O, Baliga NS, Wang JT, Ramage D, Amin N, Schwikowski B and Ideker T: Cytoscape: A software environment for integrated models of biomolecular interaction networks. Genome Res 13: 2498-2504, 2003.

21. Livak KJ and Schmittgen TD: Analysis of relative gene expression data using real-time quantitative PCR and the 2(-Delta Delta C(T)) method. Methods 25: 402-408, 2001.

22. Britton RS and Bacon BR: Intracellular signaling pathways in stellate cell activation. Alcohol Clin Exp Res 23: 922-925, 1999.

23. Zhao XK, Yu L, Cheng ML, Che P, Lu YY, Zhang Q, Mu M, Li H, Zhu LL, Zhu JJ, et al: Focal adhesion kinase regulates hepatic stellate cell activation and liver fibrosis. Sci Rep 7: 4032, 2017.

24. Wang Y, Ma J, Chen L, Xie XL and Jiang H: Inhibition of focal adhesion kinase on hepatic stellate-cell adhesion and migration. Am J Med Sci 353: 41-48, 2017.

25. Tsuchida T and Friedman SL: Mechanisms of hepatic stellate cell activation. Nat Rev Gastroenterol Hepatol 14: 397-411, 2017.

26. Gantier MP, McCoy CE, Rusinova I, Saulep D, Wang D, Xu D, Irving AT, Behlke MA, Hertzog PJ, Mackay F and Williams BR: Analysis of microRNA turnover in mammalian cells following Dicer1 ablation. Nucleic Acids Res 39: 5692-5703, 2011.

27. Weber JA, Baxter DH, Zhang S, Huang DY, Huang KH, Lee MJ, Galas DJ and Wang K: The microRNA spectrum in 12 body fluids. Clin Chem 56: 1733-1741, 2010

28. Lee HM, Nguyen DT and Lu LF: Progress and challenge of microRNA research in immunity. Front Genet 5: 178, 2014.

29. Shenoy A and Blelloch RH: Regulation of microRNA function in somatic stem cell proliferation and differentiation. Nat Rev Mol Cell Biol 15: 565-576, 2014.

30. Bartel DP: MicroRNAs: Genomics, biogenesis, mechanism, and function. Cell 116: 281-297, 2004.

31. Anthony PP, Ishak KG, Nayak NC, Poulsen HE, Scheuer PJ and Sobin LH: The morphology of cirrhosis. Recommendations on definition, nomenclature, and classification by a working group sponsored by the World Health Organization. J Clin Pathol 31: 395-414, 1978

32. Zhang CY, Yuan WG, He P, Lei JH and Wang CX: Liver fibrosis and hepatic stellate cells: Etiology, pathological hallmarks and therapeutic targets. World J Gastroenterol 22: 10512-10522, 2016.

33. Li D, He L, Guo H, Chen $\mathrm{H}$ and Shan H: Targeting activated hepatic stellate cells (aHSCs) for liver fibrosis imaging. EJNMMI Res 5: 71, 2015.

34. Trautwein C, Friedman SL, Schuppan D and Pinzani M: Hepatic fibrosis: Concept to treatment. J Hepatol 62 (Suppl 1): S15-S24, 2015.

35. Mallat A and Lotersztajn S: Reversion of hepatic stellate cell to a quiescent phenotype: From myth to reality? J Hepatol 59: 383-386, 2013.

36. Hattori S, Dhar DK, Hara N, Tonomoto Y, Onoda T, Ono T, Yamanoi A, Tachibana $M$, Tsuchiya $M$ and Nagasue $N$ : FR-167653, a selective p38 MAPK inhibitor, exerts salutary effect on liver cirrhosis through downregulation of Runx2. Lab Invest 87: 591-601, 2007.

37. Furukawa F, Matsuzaki K, Mori S, Tahashi Y, Yoshida K, Sugano Y, Yamagata H, Matsushita M, Seki T, Inagaki Y et al: p38 MAPK mediates fibrogenic signal through Smad3 phosphorylation in rat myofibroblasts. Hepatology 38: 879-889, 2003.

38. Fernandez-Ramos D, Fernandez-Tussy P, Lopitz-Otsoa F, Gutiérrez-de-Juan V, Navasa N, Barbier-Torres L, Zubiete-Franco I, Simón J, Fernández AF, Arbelaiz A, et al: MiR-873-5p acts as an epigenetic regulator in early stages of liver fibrosis and cirrhosis. Cell Death Dis 9: 958, 2018.

39. Zhou L, Liu S, Han M, Ma Y, Feng S, Zhao J, Lu H, Yuan X and Cheng J: miR-185 inhibits fibrogenic activation of hepatic stellate cells and prevents liver fibrosis. Mol Ther Nucleic Acids 10: 91-102, 2018

40. Tao L, Xue D, Shen D, Ma W, Zhang J, Wang X, Zhang W, Wu L, Pan K, Yang Y, et al: MicroRNA-942 mediates hepatic stellate cell activation by regulating BAMBI expression in human liver fibrosis. Arch Toxicol 92: 2935-2946, 2018. 
41. Hyun J, Park J, Wang S, Kim J, Lee HH, Seo YS and Jung Y: MicroRNA expression profiling in $\mathrm{CCl}(4)$-induced liver fibrosis of Mus musculus. Int J Mol Sci 17: E961, 2016.

42. Chen W, Zhao W, Yang A, Xu A, Wang H, Cong M, Liu T, Wang P and You H: Integrated analysis of microRNA and gene expression profiles reveals a functional regulatory module associated with liver fibrosis. Gene 636: 87-95, 2017.

43. Murakami Y, Toyoda H, Tanaka M, Kuroda M, Harada Y, Matsuda F, Tajima A, Kosaka N, Ochiya T and Shimotohno K: The progression of liver fibrosis is related with overexpression of the miR-199 and 200 families. PloS One 6: e16081, 2011.
44. Zhu J, Zhang Z, Zhang Y, Li W, Zheng W, Yu J, Wang B, Chen L, Zhuo Q, Chen L, et al: MicroRNA-212 activates hepatic stellate cells and promotes liver fibrosis via targeting SMAD7. Biochem Biophys Res Commun 496: 176-183, 2018.

c) (i) (9) This work is licensed under a Creative Commons CY NG ND Attribution-NonCommercial-NoDerivatives 4.0 International (CC BY-NC-ND 4.0) License. 